\title{
Bosons confined in optical lattices: The numerical renormalization group revisited
}

\author{
Lode Pollet, ${ }^{*}$ Stefan Rombouts, and Kris Heyde \\ Subatomic and Radiation Physics Department, Proeftuinstraat 86, 9000 Gent, Belgium \\ Jorge Dukelsky \\ CSIC, Serrano 12328006 Madrid, Spain \\ (Received 30 October 2003; published 1 April 2004)
}

\begin{abstract}
A Bose-Hubbard model, describing bosons in a harmonic trap with a superimposed optical lattice, is studied using a fast and accurate variational technique $(\mathrm{MF}+\mathrm{NRG})$ : the Gutzwiller mean-field (MF) ansatz is combined with a numerical renormalization group (NRG) procedure in order to improve on both. Results are presented for one, two, and three dimensions, with particular attention to the experimentally accessible momentum distribution and possible satellite peaks in this distribution. In one dimension, a comparison is made with exact results obtained using stochastic series expansion.
\end{abstract}

DOI: 10.1103/PhysRevA.69.043601

PACS number(s): 03.75.Hh, 05.30.Jp, 67.40.Db, 73.43.Nq

\section{INTRODUCTION}

The recent experiments by Greiner et al. [1] on bosons confined in an optical lattice demonstrated the transition between a Mott phase and a superfluid phase (SF), as was first predicted by Jaksch et al. [2]. The experiments are adequately described by a single band Bose-Hubbard Hamiltonian with on-site repulsion only. This type of repulsion leads to two different phases. A Mott insulating phase can exist at commensurate fillings, with a quantum phase transition to a superfluid as the density is shifted or the interaction strength weakened. In the experiments however, the quadratic confining potential adds a new term to the Hamiltonian that cuts off any long-range correlations, but Mott and superfluid regions can still occur. The experiments led to a complete revival of interest in the bosonic model, thanks to the unprecedented control over the physical parameters compared to former realizations.

Other experimental realizations of bosonic lattice systems include ${ }^{4} \mathrm{He}$ on graphite [3], superconducting islands or grains connected by Josephson junctions [4]. In this case, Cooper paired fermions are considered as bosons, at least approximately. Recently, attempts have been made to investigate at what scales the fermionic nature of paired fermions can play a role [5], and the result is that for the energy scales considered here, individual atoms can safely be described by a one-boson operator $b^{\dagger}$, as expected.

Since the work by Fisher et al. [6], the determination of the ground-state phase diagram of the Bose-Hubbard model with on-site repulsion only has attracted a lot of attention. Analytic studies using mean-field theory [6-8] and renormalization group techniques [6] led to a deeper physical understanding of the model. Strong coupling expansions $[9,10]$ gave a better quantitative picture, while quantum Monte Carlo simulations [11-15] were carried out in one and two dimensions. The one-dimensional case was recently investigated using the density-matrix renormalization group

\footnotetext{
*Electronic address: Lode.Pollet@UGent.be
}

(DMRG) [16,17], yielding the most accurate results at present. Longer-range interactions can cause charge density wave, stripe or even supersolid order [17-19]. Disorder and impurities can have dramatic effects $[6,11,17,20-22]$ and lead to even other phases. The model with a quadratic confining potential [2] has been addressed in one dimension [23] and for a small lattice in three dimensions [24], using quantum Monte Carlo methods.

In view of the enormous success of DMRG [25] in bosonic [17] and fermionic [26] real-space lattice models in one dimension, DMRG has been extended beyond these models, towards applications in metallic grains [27], quantum chemistry [28,29], and first attempts have even been undertaken towards applications in nuclear physics [30]. Unfortunately, an exact DMRG study in all three dimensions of the Bose-Hubbard model is not feasible with current computer power. DMRG was a substantial improvement [25] on the older numerical renormalization group (NRG), which had only a poor reputation in dealing with long-range interactions between fermions $[31,32]$, but we found it useful for bosonic systems (see also Ref. [33]).

The basic philosophy of this work consists of extending one-site mean-field theory to larger blocks, following the idea of the renormalization group method. A fully variational method is obtained which incorporates correlations beyond mean-field at low computational cost and which is able, unlike NRG, to accurately describe the different phases of the Mott-SF transition. We judged the computational cost as primordial, so that extensions to large lattices are in reach and so that a direct simulation of the experimental parameters can be accomplished, while the computational uncertainties remain well within the experimental uncertainty range. A disadvantage of the method is the breaking of number conservation during the intermediate steps of the renormalization procedure. In the final step particle number should be restored in principle, but this restoration is only partial when an insufficient amount of states are kept during the model space truncation. The quantity that directly relates to experiment is the momentum distribution. It was argued in Ref. [24] that the appearance of satellite peaks in the momentum distribution signals the appearance of a Mott region in the 
center of the trap. We will especially focus on the momentum distribution and on the issue of the satellite peaks.

The organization of the paper is as follows. In Sec. II we explain the method, in Sec. III we compare it to exact diagonalization methods for small latices, and in Sec. IV we present results in one, two and three dimensions. We end with the conclusion and the acknowledgments.

\section{A NUMERICAL RENORMALIZATION GROUP METHOD}

Bosons in an optical lattice realize a Bose-Hubbard Hamiltonian [2] and more specifically, we consider here the soft-core Bose-Hubbard Hamiltonian in the grand-canonical ensemble in $d$ dimensions and subject to a confining field,

$$
H=-t \sum_{\langle i, j\rangle} b_{i}^{\dagger} b_{j}+\frac{1}{2} U \sum_{i} n_{i}\left(n_{i}-1\right)+\sum_{i}\left(\epsilon_{i}-\mu\right) n_{i} .
$$

The sum $\langle i, j\rangle$ is over the nearest neighbors only. The operator $b_{i}^{\dagger}$ creates a boson on lattice site $i$ while $b_{i}$ removes it. The operator $n_{i}$ counts the local density on site $i$. The operator $N$ denotes the total number operator, $N=\Sigma_{i} n_{i}$. We take the distance $a$ between adjacent sites equal to $a=1$. The confining field acts as a local site dependent chemical potential $\epsilon_{i}$ that can be added to the chemical potential $\mu$ to form $\mu_{i}$ $=\epsilon_{i}-\mu$. In case of a site dependent $\mu_{i}$ we speak of the inhomogeneous or confined model, in case of a uniform $\mu$ we speak of the unconfined or homogeneous model. We also define the coordination number $z=2 d$ as the number of neighbors of each site and consider a linear, square, or cubic lattice of length $L$ along each axis. The energy scale is set by setting $t=1$.

This Hamiltonian is the easiest bosonic model in which two different effects compete: the kinetic energy is diagonal in momentum space and tries to delocalize the particles over the sites, while the potential energy is diagonal in coordinate space and localizes the particles.

We first discuss the physics of the model in absence of disorder in one dimension [6,34]. When the potential energy dominates, the system forms a Mott insulating phase at integer densities, which remain pinned at these integer values and the phase is incompressible. The Mott lobes are surrounded by compressible SF phases, where the densities fluctuate. This is vizualized in the mean-field phase diagram Fig. 1, which can easily be calculated [6,34]. Note that this phase diagram is approximate, in the true phase diagram the $n=1$ lobe, e.g., extends to smaller values of $U$ and the lobe closes in a pointlike fashion. There are two different phase transitions possible. When keeping the density constant at an integer value, phase fluctuations dominate and the transition is of the Berezinskii-Kosterlitz-Thouless (BKT) type. This transition can only occur at the tip of the insulator lobe, which as a consequence closes in the pointlike fashion. The generic phase transition is driven by density fluctuations and belongs to a different universality class. For a general $d$ dimension, the BKT transition generalizes to the $(d+1)$-dimensional XY universality class.

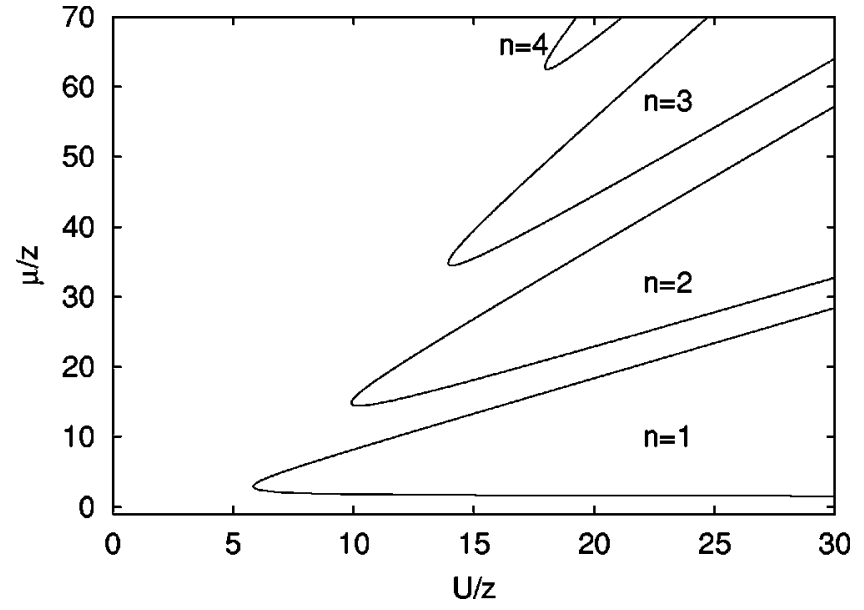

FIG. 1. The mean-field phase diagram in the units we adopt throughout the paper. The Mott lobes are indicated and surrounded by a superfluid.

A first approximation for the Bose-Hubbard model is the Gutzwiller variational ansatz $[7,8]$ leading to a decoupling of the individual lattice sites and to a mean-field theory (MF). This assumption can be described by the following substitution:

$$
b_{i}^{\dagger} b_{j} \Rightarrow \psi_{j} b_{i}^{\dagger}+\psi_{i}^{*} b_{j}-\psi_{j} \psi_{i}^{*},
$$

with $\psi_{i} \equiv\left\langle b_{i}\right\rangle$. This leads to a model where particle number symmetry can be broken, and that can exhibit a superfluid and a Mott-insulator behavior.

In order to obtain a higher accuracy, we extend the MF approximation to a renormalization group procedure by taking more correlations into account. It works as follows. Just as in MF, first break down the entire lattice to single sites and solve the problem for each site separately. The Hilbert space is truncated so that only a few basis states are kept on every site. In NRG this state selection is based on energy solely, meaning that we keep the $N_{s}$ eigenstates corresponding to the $N_{s}$ lowest-energy eigenvalues. The two sites are combined now to form a small block. At this stage, the MF approximation (2) for the hopping term between the two sites can be canceled by adding a term $\left(\psi_{i}^{*}-b_{i}^{\dagger}\right)\left(\psi_{j}-b_{j}\right)$, after which the Hamiltonian for the two sites becomes

$$
H_{12}=H_{1}+H_{2}+\sum_{i \in 1, j \in 2}\left(\psi_{i}^{*}-b_{i}^{\dagger}\right)\left(\psi_{j}-b_{j}\right)+\text { H.c. }
$$

Here, $H_{1}$ and $H_{2}$ denote the Hamiltonians of the left and right sites, respectively, and the sum runs over adjacent sites that each belong to a different block. In this first step, just the two sites 1 and 2 are meant. The Hamiltonian $H_{12}$ is diagonalized in the space spanned by the product states, which are constructed from the individual basis states of each site. After diagonalization, only a few states are kept again. Physical observables require now a rotation, since we have performed a basis rotation. The procedure repeats itself: the small blocks can be joined to form larger blocks which will themselves be the building blocks of still larger 
blocks etc. This procedure is very similar to DMRG [25]. The main differences are that in DMRG the selection of the states is based on the eigenvalues of the density matrix instead of on the lowest-energy values, and second that in NRG one combines blocks (exploiting symmetry), while in DMRG one extends the blocks site by site. In NRG one performs one calculation till the lattice is entirely built up, while in DMRG one sweeps again through the lattice till convergence is obtained. DMRG yields results with a higher accuracy, but its computational time and memory cost requirements are beyond current computer power for dimensions higher than one.

A new idea is that we improve on the standard NRG procedure by adding source terms to the Hamiltonian on the edges of the blocks. These terms compensate for the interaction with the other blocks in a mean-field way. In this way, the Hamiltonian of the local block feels already an average contribution of the blocks that have not yet accounted for, and that have a nonlocal influence on the block under consideration. After the two joining blocks are taken together, these terms need to be extracted again. If it were possible to work in an infinite Hilbert space the net effect of these source terms would be zero, but in a truncated space the calculation will depend on the values of these terms. For example, suppose we are looking for a Mott phase and these source terms are set to finite values, then we will not find the Mott phase if the source term yields contributions to states higher than the cutoff. This surely will be the case near the boundary of the Mott lobe in a homogeneous system. We have also tried to apply improved periodic boundary conditions by use of such source terms, contrary to DMRG where one usually adopts open boundary conditions. (In general, periodic boundary conditions are easier for finite-size scaling.) We will come back to the issue of source terms in the following section.

Some operators, such as the total number operator squared $N^{2}$ also acquire a contribution from the cross terms between the two building blocks. So, more than a simple rotation is needed in this case, and contributions from the total number operator $N$ must be taken into account. Schematically, $\left\langle N_{12}^{2}\right\rangle=\left\langle N_{1}^{2}\right\rangle+\left\langle N_{2}^{2}\right\rangle+2\left\langle N_{1} N_{2}\right\rangle$, in which the indices 1,2 indicate the two joining blocks.

One can obtain the one-body density matrix in coordinate space with the MF+NRG method, but at a low accuracy because correlations $b_{i}^{\dagger} b_{j}$ are inaccurate when $i$ is not the first site of the renormalization procedure, and we need the entire matrix for a confined system. However, we are able to directly calculate the diagonal of the momentum density operator $\rho_{k, k} \equiv \rho_{k}$ (in one dimension),

$$
\begin{aligned}
\rho_{k} & =\sum_{i, j} e^{-i k(i-j)} b_{i}^{\dagger} b_{j} \\
& =\sum_{i \in L, j \in R} \cos [k(i-j)] b_{i}^{\dagger} b_{j} .
\end{aligned}
$$

Here we sum over all sites of the blocks, easing the problem encountered with the one-body density matrix in coordinate space. This operator can be rewritten as

$$
\begin{aligned}
\rho_{k}= & \sum_{i \in L, j \in R} \cos (k i) \cos (k j) b_{i}^{\dagger} b_{j}+\sin (k i) \sin (k j) b_{i}^{\dagger} b_{j} \\
= & \left(\sum_{i \in L} \cos (k i) b_{i}^{\dagger}\right)\left(\sum_{j \in R} \cos (k j) b_{j}\right)+\left(\sum_{i \in L} \sin (k i) b_{i}^{\dagger}\right) \\
& \times\left(\sum_{j \in R} \sin (k j) b_{j}\right) \\
= & C_{k_{L}}^{\dagger} C_{k_{R}}+S_{k_{L}}^{\dagger} S_{k_{R}} .
\end{aligned}
$$

Hence we have to keep track of linear combinations of the creation $c_{i}^{\dagger}$ and annihilation $c_{i}$ operators. The parts in which both sites $i$ and $j$ belong to the same block [left $(L)$ or right $(R)]$ have been omitted, since their updating consists only of a rotation to the newly truncated basis. When the sites belong to different blocks, there is also a contribution of the cross term just as with the operator $N^{2}$, but it still suffices to update the $C$ and $S$ operators. The extension to higher dimensions of Eq. (5) is straightforward. We normalize the Fourier transform by adding prefactors $1 / L$ so that the trace of the density matrix in momentum space yields the number of particles in the system.

\section{COMPARING THE METHOD WITH EXACT RESULTS FOR SMALL LATTICES}

In this section we consider a small lattice in one dimension and in the absence of any kind of disorder.

We have checked the code by comparing the resulting energies to direct Lanczos diagonalization values for a lattice containing eight sites in one dimension. The one-dimensional Bose-Hubbard model with periodic boundaries is a worstcase scenario for our MF+NRG procedure. The results are summarized in Table I. The parameters in the table vary from a SF phase to a Mott phase. As expected, very deep in a Mott phase or in a SF phase we obtain a very good accuracy. Note that the Lanczos diagonalization was performed with a fixed

TABLE I. Comparison of the energies $\left(E_{16}\right.$ and $\left.E_{32}\right)$ per site obtained by the MF+NRG method for a modest number of states (16 and 32) kept after each diagonalization with the results $E_{L}$ of a Lanczos diagonalization procedure for a small lattice of eight sites in one dimension. The deviations $\left(D_{16}\right.$ and $\left.D_{32}\right)$ are indicated and can be made smaller by keeping more states after each diagonalization. The mean-field values are in the last column.

\begin{tabular}{lccccccc}
\hline \hline$U$ & $\mu$ & $E_{L}$ & $E_{16}$ & $D_{16}(\%)$ & $E_{32}$ & $D_{32}(\%)$ & $E_{M F}$ \\
\hline 2.0 & -0.5 & -1.359 & -1.337 & 1.57 & -1.347 & 0.91 & -1.24 \\
4.0 & 0.7 & -0.932 & -0.901 & 3.38 & -0.914 & 1.99 & -0.78 \\
6.0 & 1.8 & -0.656 & -0.612 & 6.65 & -0.635 & 3.15 & -0.44 \\
8.0 & 2.5 & -0.494 & -0.467 & 5.60 & -0.483 & 2.40 & -0.27 \\
10.0 & 3.5 & -0.397 & -0.382 & 3.53 & -0.392 & 1.09 & -0.10 \\
12.0 & 4.0 & -0.331 & -0.324 & 2.35 & -0.330 & 0.31 & 0.00 \\
14.0 & 5.0 & -0.284 & -0.283 & 0.50 & -0.284 & 0.23 & 0.00 \\
16.0 & 6.0 & -0.249 & -0.247 & 0.38 & -0.248 & 0.07 & 0.00 \\
18.0 & 7.0 & -0.221 & -0.221 & 0.30 & -0.222 & 0.04 & 0.00 \\
20.0 & 15.2 & -0.199 & -0.181 & 8.70 & -0.199 & 0.17 & 0.00 \\
\hline \hline
\end{tabular}




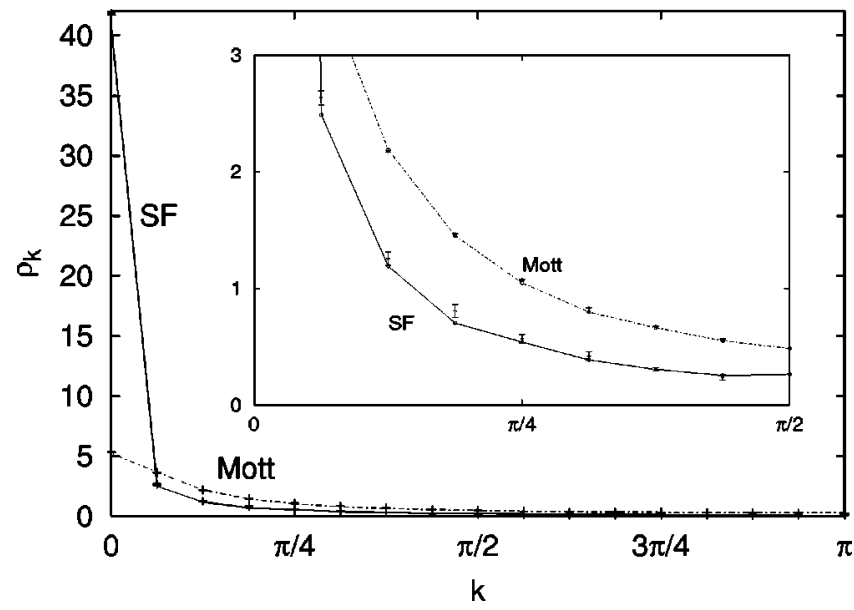

FIG. 2. Checking the momentum distribution obtained with $\mathrm{MF}+\mathrm{NRG}$ to a SSE calculation for an unconfined system in one dimension of 32 sites. Calculations have been done for a system in the Mott phase ( $U=6, \mu=2$, dashed line) and for a system in the SF phase ( $U=2, \mu=1$, full line). The errors on the SSE data points are shown but very small. In the inset, the MF+NRG data points are indicated explicitly by small circles, while "+" signs with error bars indicate the SSE data points.

boson number, while in the $\mathrm{MF}+\mathrm{NRG}$ we adjusted the chemical potential in order to fix the density. The deviations should be interpreted accordingly.

We have also checked observables like the local density $n_{i}$ and local compressibility $\kappa_{i}$ against results obtained with the stochastic series quantum Monte Carlo [35] (SSE) method for larger lattices. Because the calculation of the momentum distribution seems most critical, we have explicitly shown in Fig. 2 the good agreement between the calculation of the momentum distribution with the renormalization group and the SSE method for a SF and a Mott phase. The one-body density matrix with the SSE method has been obtained by applying the idea of Ref. [36] to soft-core bosons. The SSE method served as a testing ground for the renormalization method here. So we have shown that a lot of physics might be examined with our method.

The parameter $N_{s}$ that fixes how many states are kept in the truncation determines the accuracy of the results. As we have seen in Table I energies can decrease by increasing $N_{s}$, while for observables like the local density the fluctuations become smaller. We have examined how the grand-canonical potential $\Phi$ decreases when $N_{s}$ is increased in the upper part of Fig. 3 for a system of $L=1024$ sites (so that finite-size effects can be filtered out of this discussion) in the SF phase but very close to the generic Mott phase transition. This corresponds to the worst case scenario for our method. Inclusion of just a few states leads to a rapid decrease in the grandcanonical potential, but once more than 20 states are kept, the potential decreases only very gradually. The exact result $\Phi / L=-1.917(2)$ in Fig. 3 was again obtained by the SSE method, while with $N_{s}=40$ we reached $\Phi / L=-1.90$. Without source terms, we found that the calculated average grandcanonical potential per site was $\Phi / L=-1.87$ with $N_{s}=40$, giving further evidence of the usefulness of the source terms. It is the sweeping property of the DMRG algorithm that
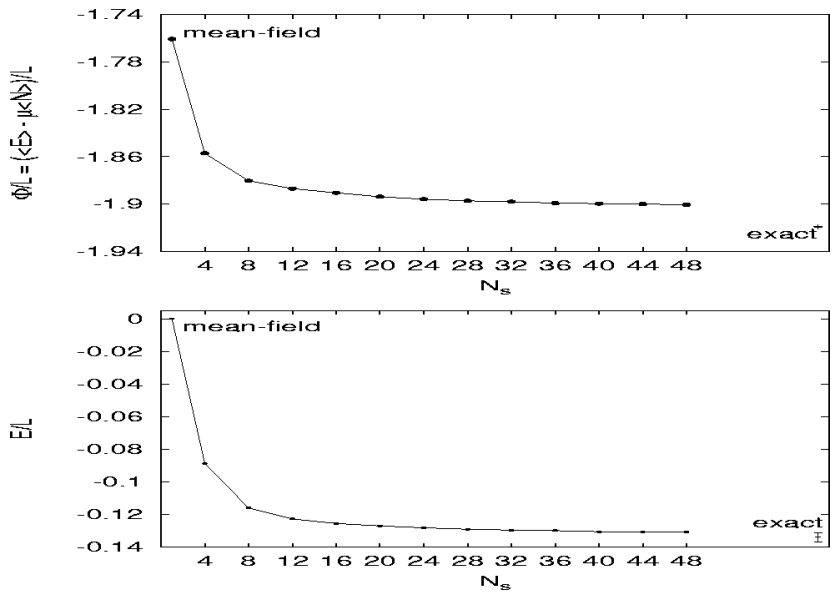

FIG. 3. Upper: The system is variational in the average grandcanonical potential per site $\Phi / L=(\langle E\rangle-\mu\langle N\rangle) / L$. As the number of kept states $N_{s}$ is increased, the grand-canonical potential decreases. The full line is a guide to the eye. The parameters are chosen such that the system is near a generic phase transition on the SF side ( $U=4, \mu=1, L=1024)$. This corresponds to the worst case scenario for our method. The mean-field $\left(N_{s}=1\right)$ and exact (corresponding to $N_{s}=\infty$ ) results are indicated. Lower: The exponential convergence of the energy is shown. The system is deep in the Mott phase with parameters $U=30, \mu=16$, and $L=1024$.

could improve the results here substantially, something we tried to avoid from the onset since this property is computationally too costly in higher dimensions. The discrepancy with the exact result in Fig. 3 reduces rather slowly at higher values of $N_{s}$, primarily because of the effects of block extension (reflected in the curves of the local densities and local energies in Fig. 4 for the same effect) and complications due to the periodic boundary conditions. The exact result should be recovered in the limit of $N_{s}$ equal to the dimension of the Fock space for each block. In addition, for a system that is already deep in one of both phases, the MF+NRG method converges very rapidly to the exact result, as the energy curve shows in the lower part of Fig. 3 for a system in the Mott phase. Here, the energy and grand potential differ only by a constant.

On the other hand, the parameter $N_{s}$ also largely determines the required computer time: observables scale as $N_{s}^{2}$ per lattice site in memory cost, and the most time consuming operation is the rotation of variables, which scales as $N_{s}^{6}$ (multiplication of matrices of the order of $N_{s}^{2}$ ). All our calculations have been performed on a Pentium IV, $1.6 \mathrm{GHz}$ or a Pentium III, each with 500 MB RAM. Larger lattices and higher values of $N_{s}$ can straightforwardly be implemented on more performant hardware, but requiring that all occupation numbers of the truncated states are arbitrarily small on one hand and on the other hand wishing to study large lattices in high dimensions near a quantum phase transition is still not achievable.

One of the crucial parameters of the method is the source term that is inserted at the boundary of each block. If we set it to zero, our method reduces to the standard NRG method. It can be seen in Fig. 4 that the fluctuations can be damped much better in a SF phase if we set the source term equal to 

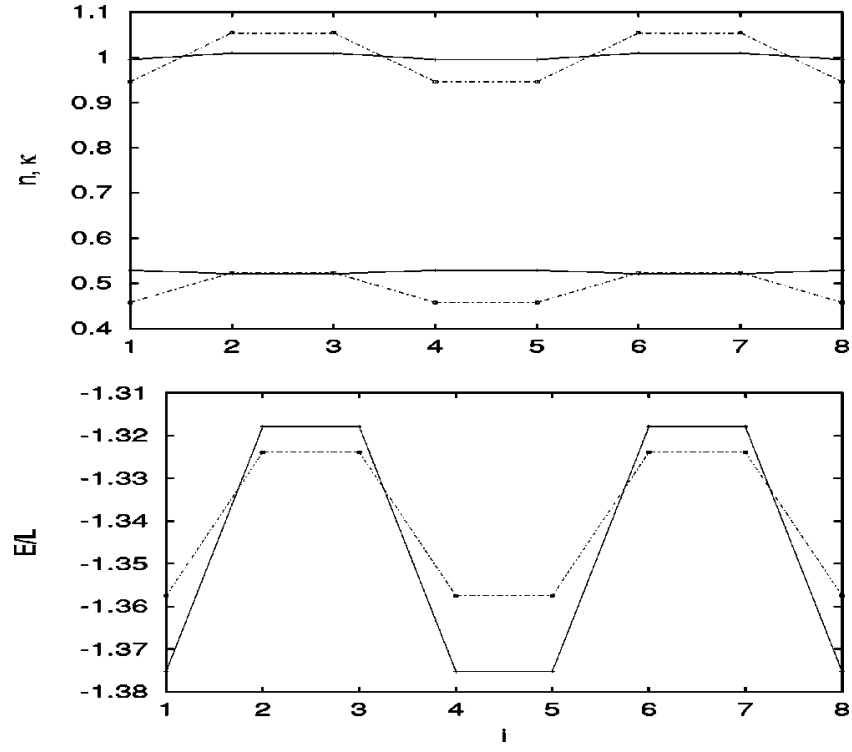

FIG. 4. The figure shows how source terms can improve the calculations. Local energy per site $E_{L}$, local compressibility $\kappa_{i}$, and local density $n_{i}$ from bottom to top are plotted as a function of the lattice index $i$ for a homogeneous model of eight sites in a SF phase ( $U=2, \mu=-0.5$, the same values as the upper row in Table I). The dashed line has source terms set to zero, while for the full line they are set to their mean-field values.

the MF expectation value of the operator $c_{i}$, while the total energy deviates now $0.9 \%$ from to the exact result instead of $1.4 \%$ without the source terms. For a homogeneous model in the thermodynamic limit, the value of $\left\langle c_{i}\right\rangle$ should be siteindependent and the source terms should be chosen equal as well.

As argued in the preceding section, the source terms are optional and need to be chosen carefully. It is well known [34] that a Mott phase can only be found if $[H, N]=0$. Source terms might violate this condition near a Mott-SF transition. The addition of source terms might lead to an incorrect prediction of a SF phase when the compensation of the source terms in the renormalization scheme is not complete. The source terms could yield contributions to states that are thrown away after truncation of the Hilbert space and these contributions can be quite large when the parameter $N_{s}$ is chosen too low. This might lead to an incorrect value of the transition point. In addition, even if the transition point of a generic Mott-SF phase transition was known exactly, and we would study the SF side of this transition, the source terms should still be chosen carefully. This can be understood as follows: any net contribution of a source term will deal with long-range correlations in the same way as mean-field does, and we know that the correlators predicted by mean-field are only valid in $d=3$ dimensions. For example, the density is a valid order parameter for the generic Mott-SF transition [34], with

$$
\begin{gathered}
n \sim \mu^{1 / 2} \quad d=1, \\
n \sim \mu \quad d=3 .
\end{gathered}
$$

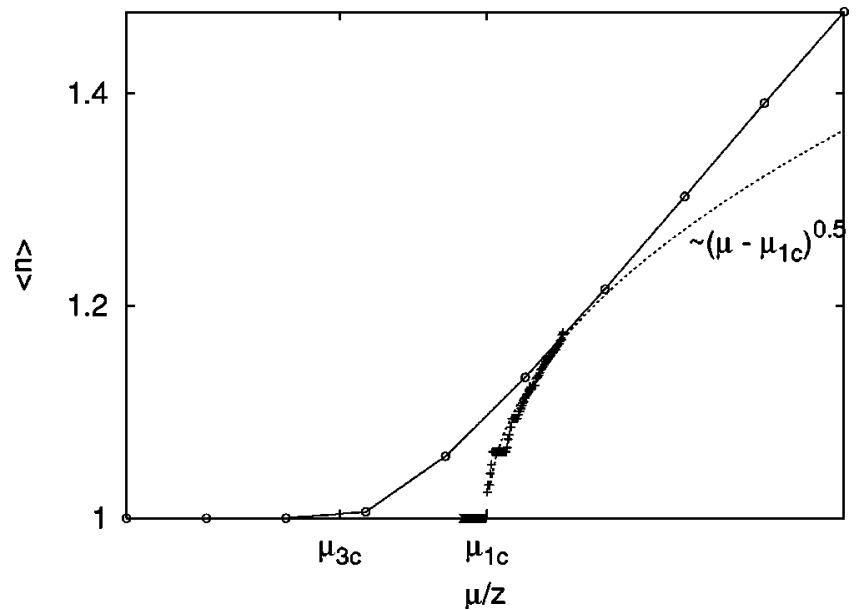

FIG. 5. Evolution of the density in the neighborhood of the generic phase transition between the Mott and SF phase for one ("+" marks) and three (empty circles) dimensions. The dashed line is a fit according to Eq. (6). In these calculations the source terms are set to zero and lattices of size $L=1024$ sites were studied. Inclusion of the source terms in one dimension would lead to a similar plot as in the $3 \mathrm{D}$ case.

Briefly said, the source terms would in one and two dimensions lead to an improved mean-field theory, in the sense that the correlators would approximately have the same exponents as in mean-field theory and the Mott lobe would extend a little bit farther into parameter space. This is also explained in Fig. 5. When $N_{s}$ is large enough, these possible dangers become less severe. In the confined case, any longrange correlations are effectively cut off and the addition of source terms is always expected to improve the calculations, as has been verified.

It was also tempting to study what happens if the blocks were extended by a single site only. That leads to the same number of diagonalizations but more rotations are needed. For the unconfined case, this yielded quite good results, often smoother than in the block renormalization case. However, for the confined case this procedure did not produce regions with integer density and should hence only be used with great care.

The MF+NRG procedure also offers a substantial improvement over MF results. The MF transition between the SF phase and the Mott phase is independent of the dimension of the system and is located at $U_{c} \approx 5.83$, while a DMRG [17] study locates it at $U_{c} / z \approx 3.36$ in one dimension and a strong-coupling expansion [10] locates it at $U_{c} / z \approx 4.18$ in two dimensions. We have performed a simulation at $U / z$ $=5.0$ in one, two, and three dimensions. While MF theory predicts a SF phase (calculation yields $\langle c\rangle=0.496$ ) the true phase should be a Mott phase in one and two dimensions and we even found a Mott phase in three dimensions. These results can be seen in Fig. 6 where the local density and local compressibility are shown.

\section{RESULTS}

Now that we have critically examined the approximations made in the $\mathrm{MF}+\mathrm{NRG}$, we apply it to parameter regions 


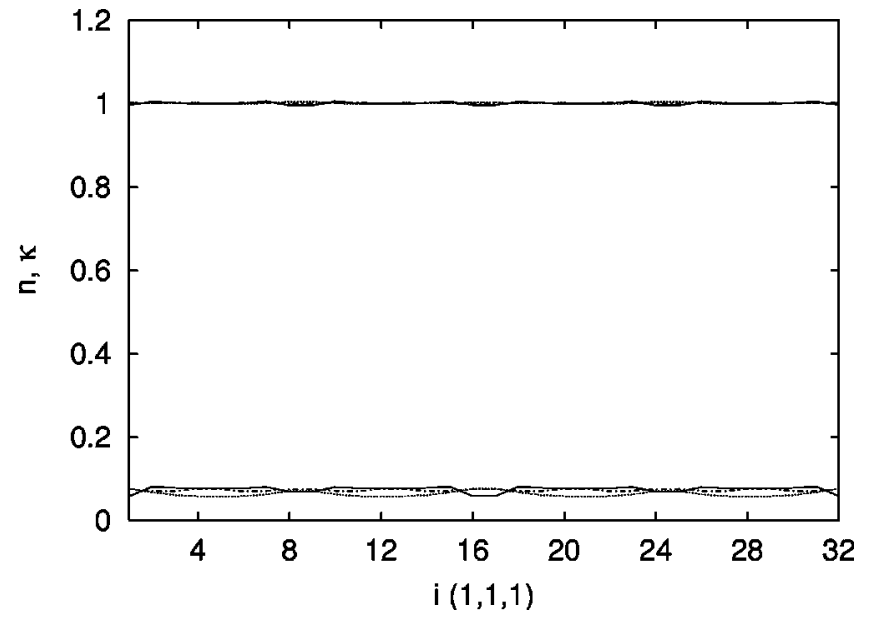

FIG. 6. Local density (upper curves) and local compressibility (lower curves) for parameters $U / z=5.0$ and $\mu / z=2.0$ in one (full line), two (dotted line), and three (dashed line) dimensions. MF theory predicts a SF phase, while the true phase in one, two, and three dimensions is a Mott phase.

where the results are unambiguous. In all calculations the size of the lattices corresponds to the maximal achievable size, while a sufficient amount of states has been kept.

\section{A. Results in one dimension}

We have, in complete analogy with Ref. [23], confined a Bose gas in a lattice of 128 sites in a trapping potential of the form

$$
\epsilon_{i}=v_{c}(i-L / 2)^{2},
$$

with $v_{c}=0.008$. Choosing the parameter this way allows for nice fillings in the center and for densities going smoothly to zero near the edges of the trap.

In Fig. 7 we see how plateaus with local fillings of an integer number of bosons can arise as more and more particles enter the system. The global compressibility is never zero, as it is the case in the unconfined model in the thermo-

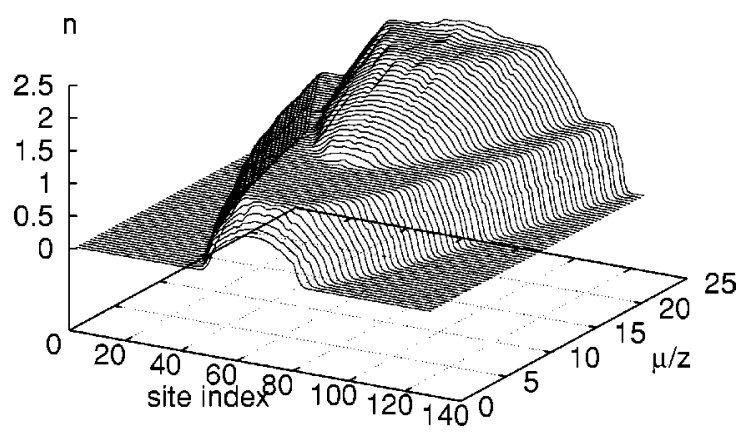

FIG. 7. Profile of the local density $n$ along the sites and as a function of the chemical potential $\mu$. The on-site repulsion is $U / 2$ $=7.1$. Above a certain value of $\mu$ we see the emergence of a plateau $(n=1)$, and when the total number of particles is even more increased, we see the reemergence of a compressible region. This happens first around the center and continues to exist till a plateau with $n=2$ is reached. These results confirm the result of Ref. [23].

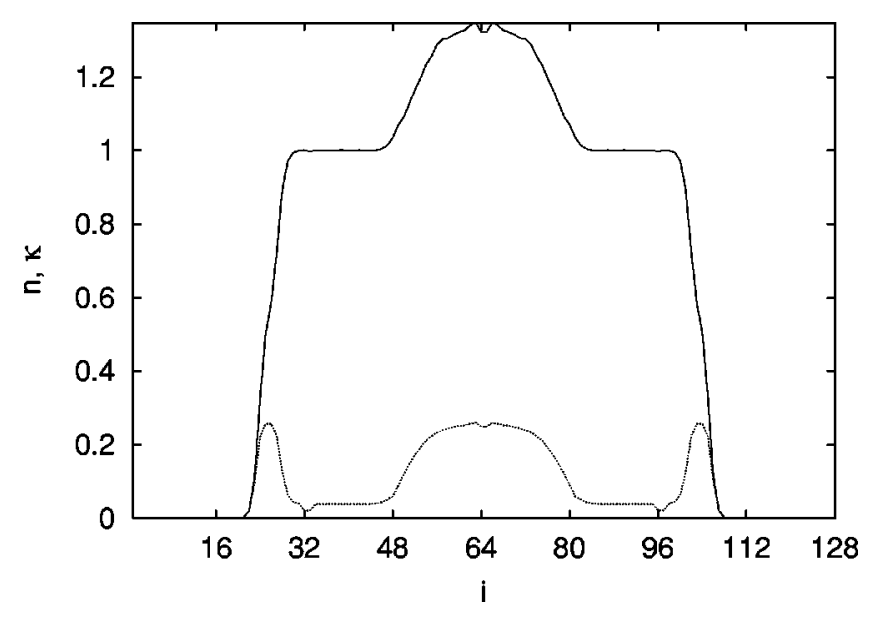

FIG. 8. Profile of the local density $n$ (solid line) and local compressibility $\kappa$ (dashed line) along the sites for $U / 2=7.1, \mu / 2$ $=6.1, L=128, v_{c}=0.008$, confirming again the result of Ref. [23].

dynamic limit. We cannot speak therefore of a true quantum phase transition, the confining potential effectively cuts off all long-range correlations. However, in local regions the local compressibility can get very low and the local density can get stuck at integer values, reflecting a local Mott region. This can be seen in Fig. 8. All these results are completely in line with those of Ref. [23]. Also, for a canonical calculation with an incommensurate filling a Mott phase with integer density can still be found, because the confining potential changes the local chemical potential.

Looking along the sites can be interpreted as different $\mu$ slices of the phase diagram in the $(U, \mu)$ plane for the unconfined model [6]. This allows to calculate the site at which a Mott domain is entered or left. It is also clear that the BKT transition has no analog in the unconfined case. We refer to Ref. [23] for a state diagram. The authors of Ref. [23] also claim that $\kappa_{i} \sim\left(n_{i}-1\right)$ as the Mott lobe is approached, independent of the on-site repulsion $U$ or the chemical potential $\mu$. In our calculations the same behavior was seen for parameters that are of the same order of magnitude, but for small and large values of $U$ the local compressibilities did not reach to the same values in the Mott region.

\section{B. Results in two dimensions}

\section{Homogeneous case}

In principle it is possible to determine the phase diagram, but a complication that makes a comparison more difficult is that in the literature [10] calculations are usually based on a fixed density while we are working in the grand-canonical ensemble. The physically most interesting case is with a disordered chemical potential [22]. A phase diagram requires a study of phase transitions and very close to a transition point it is important to include more and more states into the truncated Hilbert space and at the same time going to larger lattices. The method described here can only give a qualitative answer and is not fit to quantitatively yield the exact location of the transition point and does not allow to calculate the critical exponents in an unambiguous way. 


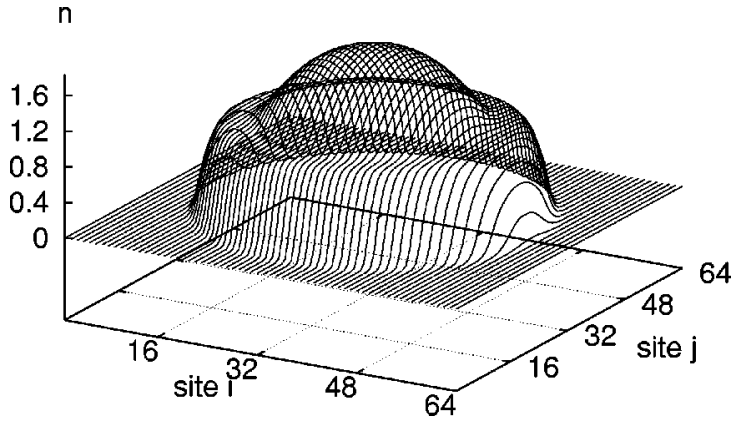

FIG. 9. Local density for a lattice consisting of $L=64 \times 64$ sites and with parameters $U=23.2, \mu=28.0, v_{c}=0.05$. Note again the region with fixed integer density and the smooth transitions.

The problem encountered here is a "memory effect" when $N_{s}$ is not high enough. When the source terms are set to zero and a large lattice of $L=256 \times 256$ is taken, a calculation with a too low $N_{s}$ predicts a Mott phase while an increased $N_{s}$ leads to a SF phase. So, starting from a Mott phase (zero source terms) results in a Mott phase and starting from a SF phase (finite source terms) reveals a SF phase. The issue of the phase diagram is very similar to the difficulties encountered with the strong-coupling expansion by Freericks and Monien [9], although their starting point is entirely different. As they point out, their method cannot describe the physics close to the tricritical point, the density fluctuations dominate even close to the tricritical point, and they can notice that the shape of the Mott lobes has changed from one to higher dimensions. Due to the limitations in our method we see the same qualitative aspects, but we ran into the same quantitative difficulties, with the same order of uncertainties. We will not report on calculations of the phase diagram here.

\section{Confined case}

The trapping potential takes on each site $i$ the value

$$
\epsilon_{i}=v_{c} r_{i}^{2}
$$

where $r_{i}$ measures the distance from the present site $i$ to the center of the trap. The same holds in three dimensions. In Fig. 9 we plot the local density for a system of $L=64 \times 64$ sites, with $U=23.2, \mu=28.0, v_{c}=0.05$ and the space is constantly truncated to 32 states, principally in line with our philosophy of a limited but fast and reliable calculation. In Fig. 10 we show the local compressibility for the same system, but only one quarter of the figure is shown. The other parts are symmetric. Note again that there exists a Mott insulating region with integer density. The transition from the SF region to this Mott region is not sharp, and the local compressibility in the Mott region is small but remains finite.

Another example can be found in Figs. 11 and 12 for a lattice of $128 \times 128$ sites, showing Mott behavior and where for a slightly weaker $U$ a new SF region would emerge in the center of the trap.

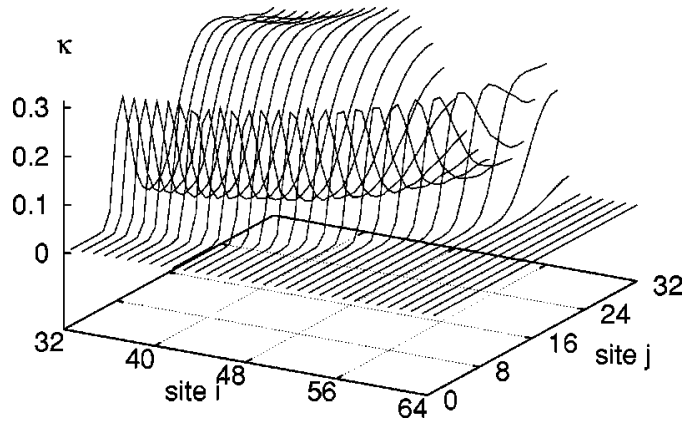

FIG. 10. Local compressibility as a function of the site indices and with the same parameters as in Fig. 9.

\section{Results in three dimensions}

The original experiments by Greiner et al. [1] were performed in three dimensions, with laser beams cutting the atomic cloud in about $L=65 \times 65 \times 65$ sites, and a local density varying around $n=2.5$ atoms per site. Mott and SF behavior were demonstrated after examination of the interference pattern of the laser images of the free expanding cloud. This means that the quantity of computational interest is the momentum distribution. In the original experimental setup, the absorption images of the three-dimensional distribution are taken along two orthogonal axes, revealing only the integral over the third direction. The observed fading of the Bragg peaks had nothing to do with the appearance of Mott behavior, and happened actually when the system was already very deep in the Mott insulating phase.

So what could be a clear signal of the transition? It was argued in Ref. [24] that satellite peaks in the momentum distribution are related to the appearance of a Mott region in the center of the trap. Once the Mott region spanned almost the entire lattice, the peaks disappeared into the typical broad, low-peaked Mott distribution. However, their worm Monte Carlo calculation was only on a lattice of $L=16$ $\times 16 \times 16$ and it can be expected that for a larger lattice the central SF peak might be so dominant that the satellite peaks can hardly be resolved. We present a calculation on a lattice of $L=32 \times 32 \times 32$. We show the momentum distribution in Fig. 13 along the $(1,0,0)$ axis, for a system with an emerging Mott region of $n=1$ in the center of the trap. As in Ref.

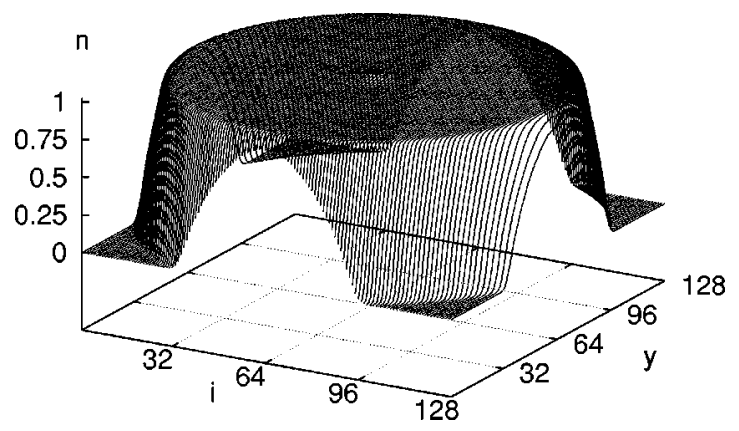

FIG. 11. Local density as a function of the site indices for a lattice of $L=128 \times 128$ sites and with parameters $U=22.0, \mu$ $=35.6, v_{c}=0.008$. The system is very close to developing a new SF peak in the center. 


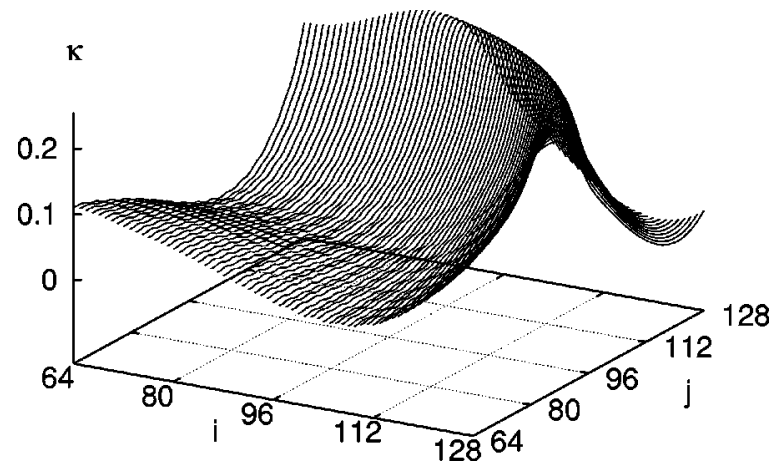

FIG. 12. Same as in Fig. 11 but for the local compressibility now. The parts of the plot that are not shown are symmetric.

[24] we see satellite peaks along the $(1,0,0)$ direction, but the central peak dominates. The satellite peaks are only $4.5 \%$ in magnitude of the central peak and will be difficult to resolve in practice. For the experimental setup with its lattice of about $L=65 \times 65 \times 65$ sites, the situation will even be worse. We also note that the satellite peaks depend on the direction of investigation, no satellite peaks were seen, e.g., along the $(1,1,1)$ direction in Fig. 13. This direction dependency is a consequence of the breaking of rotational symmetry in a finite lattice, and its effects should diminish when larger lattices are taken.

Furthermore, the average density in the experiments was about $n \approx 2.5$ in the center of the trap [1]. There are no satellite peaks when the central density is noninteger, despite a broad Mott $n=1$ region for an on-site repulsion $U$ that is strong enough. This Mott region is reflected in the tail of the momentum distribution [24]. For a system with local densities varying between $n=2$ and $n=3.2$ (all noninteger densities), we nevertheless found satellite peaks in Fig. 14, and calculations showed the same behavior for densities ranging

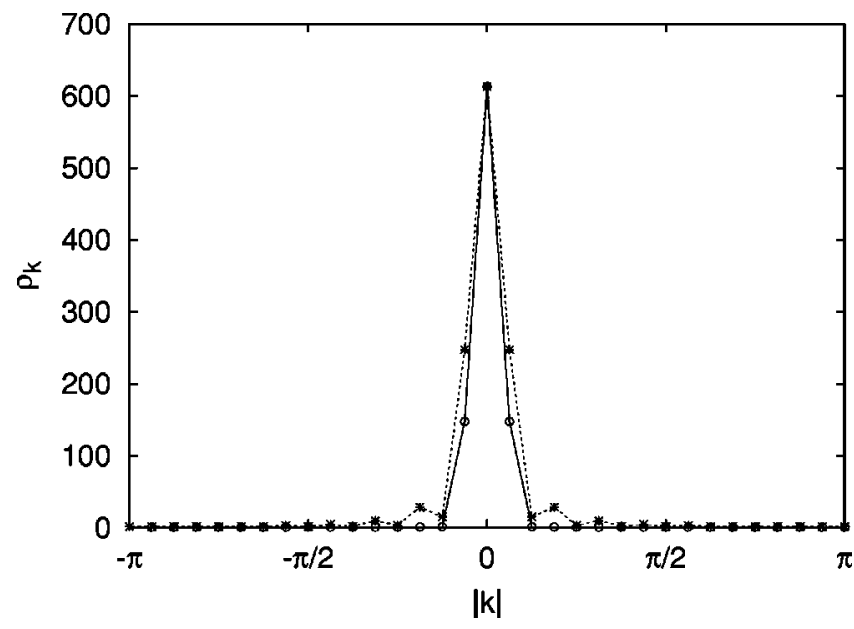

FIG. 13. Momentum distribution for a system with a Mott plateau $(n=1)$ in the center. The parameters are $U / z=6.5, \mu / z$ $=2.6, v_{c}=0.04$ and $L=32 \times 32 \times 32$. The dashed line represents the distribution along the $(1,0,0)$ direction, while the full line is taken along the $(1,1,1)$ direction. According to Ref. [24] the satellite peaks in the dashed curve point at an emerging Mott region in the center of the trap.

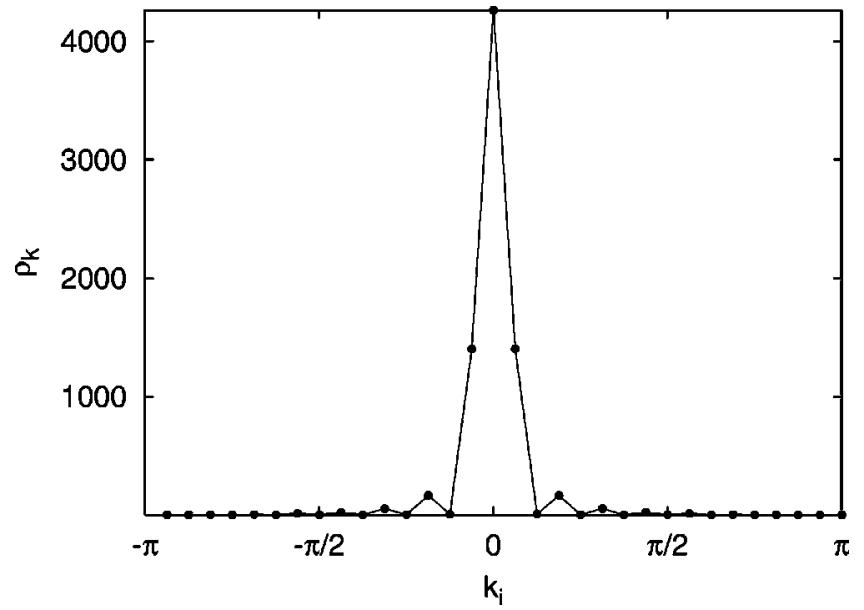

FIG. 14. Momentum distribution along the $(0,0,1)$ axis for a system with particle densities varying between $n=2$ and $n$ $=3.2\left(U / z=11, \mu / z=30, v_{c}=0.1, L=32 \times 32 \times 32\right)$. The appearance of satellite peaks cannot be related to the emergence of a Mott region in the center of the trap.

between $n=3$ and $n=4$. These peaks cannot possibly be related to the emergence of a Mott region in the center of the trap. Local densities of $n=2$ at the border of the trap cannot occur experimentally, but this situation can be thought of as the central region of a larger lattice, from which the outer regions are not trapped any more.

When going to higher values of $U$ and $\mu$, it is, in principle, possible to have Mott phases at $n=2$ and $n=3$. In the mean-field phase diagram of the homogeneous model [8], the different Mott lobes corresponding to densities $n=1, n=2$, etc. get closer to each other along the direction of the chemical potential $\mu$ (see Fig. 1). With the confining potential $\epsilon_{i}$ present, the local densities along the different sites can be interpreted as a scan of the homogeneous model [6]. Hence in a small finite lattice it is not a priori clear if there are noninteger densities between the different broad Mott regions. For a system with parameters $U / z=30, \mu / z=75, v_{c}$ $=9.1$, and $L=16 \times 16 \times 16$, we found very few noninteger densities. The density profile consisted of four plateaus with $n=0,1,2,3$, respectively. We have almost a superposition of four Mott phases leading to the momentum distribution in Fig. 15, which is very low peaked and broad. When the local particle density in the center of the trap is gradually increased from $n<3$ till the Mott region with $n=3$, and while there already exist broad Mott regions with $n=1$ and $n=2$, we did not witness any satellite peaks, because the Mott behavior of the $n=1$ and $n=2$ plateaus already dominated the momentum distribution.

We are led to the observation that it will be difficult to indicate the transition experimentally by satellite peaks, and that only examination of the intensity and the width of the central peak along one direction might be at hand to directly reveal Mott behavior.

\section{CONCLUSION}

In summary, we studied the Bose-Hubbard model subject to a confining field in the grand-canonical ensemble. We 


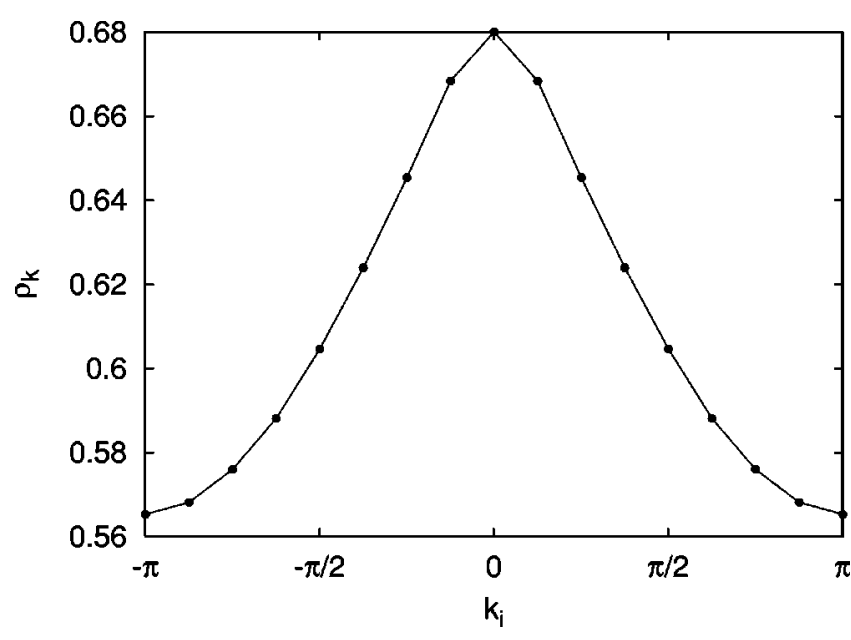

FIG. 15. Momentum distribution along the $(0,0,1)$ axis for a system with particle densities varying between $n=0$ at the edge of the trap to $n=3$ at the center $\left(U / z=30, \mu / z=75, v_{c}=9.1, L=16\right.$ $\times 16 \times 16$ ), leading to 2280 particles in the system. The distribution is broad and not peaked, signaling virtually overall Mott behavior.

combined the Gutzwiller mean-field (MF) ansatz with a numerical (block) renormalization group method (NRG) and we could calculate observables like local densities, energies, the momentum distribution, etc. The goal was to achieve variational results with energies much lower than in meanfield theory and at a low computational cost in order to make studies of large lattices in higher dimensions feasible. We have extensively discussed the advantages and limitations of this method. The inclusion of source terms on the edges of the blocks improved results in the SF phase.

We have examined the smooth transition between SF and Mott regions in the presence of a confining field. Although there is no real "order parameter" to be found in the momentum distribution, the momentum distribution can nevertheless reveal important qualitative differences between pure SF systems and systems with dominant Mott behavior. These differences can experimentally best be seen in the central peak. Possible satellite peaks might be difficult to resolve when the total number of confined particles is large and when the filling factors are not of the order of unity.

\section{ACKNOWLEDGMENTS}

The authors wish to thank I. Bloch, M. Greiner, T. Papenbrock, J. Ryckebusch, and D. Van Neck for valuable discussions. This work was supported by the Research Board of the University of Ghent and the Fund for Scientific Research, Flanders.
[1] M. Greiner, O. Mandel, T. Esslinger, T. Hänsch, and I. Bloch, Nature (London) 415, 39 (2002).

[2] D. Jaksch, C. Bruder, J. I. Cirac, C. W. Gardiner, and P. Zoller, Phys. Rev. Lett. 81, 3108 (1998).

[3] G. T. Zimanyi, P. A. Crowell, R. T. Scalettar, and G. G. Batrouni, Phys. Rev. B 50, 6515 (1994), and references therein.

[4] A. van Oudenaarden and J. E. Mooij, Phys. Rev. Lett. 76, 4947 (1996).

[5] S. Rombouts, D. Van Neck, K. Peirs, and L. Pollet, Mod. Phys. Lett. A 17, 1899 (2002).

[6] M. P. A. Fisher, P. B. Weichman, G. Grinstein, and D. S. Fisher, Phys. Rev. B 40, 546 (1989).

[7] K. Sheshadri, H. R. Krishnamurthy, R. Pandit, and T. V. Ramakrishnan, Europhys. Lett. 22, 257 (1993).

[8] D. van Oosten, P. van der Straten, and H. T. C. Stoof, Phys. Rev. A 63, 053601 (2001).

[9] J. K. Freericks and H. Monien, Europhys. Lett. 26, 545 (1994).

[10] N. Elstner and H. Monien, Phys. Rev. B 59, 12184 (1999).

[11] R. T. Scalettar, G. G. Batrouni, and G. T. Zimanyi, Phys. Rev. Lett. 66, 3144 (1991).

[12] G. G. Batrouni and R. T. Scalettar, Phys. Rev. B 46, 9051 (1992); G. G. Batrouni, R. T. Scalettar, G. T. Zimanyi, and A. P. Kampf, Phys. Rev. Lett. 74, 2527 (1995).

[13] W. Krauth and N. Trivedi, Europhys. Lett. 14, 627 (1991); W. Krauth, N. Trivedi, and D. Ceperley, Phys. Rev. Lett. 67, 2307 (1991).

[14] M. C. Cha, M. P. A. Fisher, S. M. Girvin, M. Wallin, and A. P. Young, Phys. Rev. B 44, 6883 (1991).

[15] V. A. Kashurnikov and B. V. Svistunov, Phys. Rev. B 53,
11776 (1996).

[16] T. D. Kühner and H. Monien, Phys. Rev. B 58, 14741 (1998).

[17] T. D. Kühner, S. R. White, and H. Monien, Phys. Rev. B 61, 12474 (2000).

[18] G. G. Batrouni and R. T. Scalettar, Phys. Rev. Lett. 84, 1599 (2000).

[19] A. van Otterlo and K.-H. Wagenblast, Phys. Rev. Lett. 72, 3598 (1994); A. van Otterlo, K.-H. Wagenblast, R. Baltin, C. Bruder, R. Fazio, and G. Schön, Phys. Rev. B 52, 16176 (1995).

[20] C. L. Kane and M. P. A. Fisher, Phys. Rev. B 46, 15233 (1992).

[21] T. Giamarchi and H. J. Schulz, Europhys. Lett. 3, 1287 (1987).

[22] N. Prokof'ev and B. Svistunov, Phys. Rev. Lett. 92, 015703 (2004).

[23] G. G. Batrouni, V. Rousseau, R. T. Scalettar, M. Rigol, A. Muramatsu, P. J. H. Denteneer, and M. Troyer, Phys. Rev. Lett. 89, 117203 (2002).

[24] V. A. Kashurnikov, N. V. Prokof'ev, and B. V. Svistunov, Phys. Rev. A 66, 031601(R) (2002).

[25] S. R. White, Phys. Rev. Lett. 69, 2863 (1992); Phys. Rev. B 48, 10345 (1993); S. R. White and R. M. Noack, Phys. Rev. Lett. 68, 3487 (1992).

[26] S. R. White, Phys. Rep. 301, 187 (1998).

[27] J. Dukelsky and G. Sierra, Phys. Rev. Lett. 83, 172 (1999); Phys. Rev. B 61, 12302 (2000).

[28] S. R. White and R. L. Martin, J. Chem. Phys. 110, 4127 (1998).

[29] Ö. Legeza, J. Röder, and B. A. Hess, Phys. Rev. B 67, 125114 (2003). 
[30] J. Dukelsky and S. Pittel, Phys. Rev. C 63, 061303(R) (2001); J. Dukelsky, S. Pittel, S. S. Dimitrova, and M. V. Stoitsov, ibid. 65, 054319 (2002).

[31] K. G. Wilson, Rev. Mod. Phys. 47, 773 (1975).

[32] J. W. Bray and S. T. Chui, Phys. Rev. B 19, 4876 (1979)

[33] R. Bulla, N.-H. Tong, and M. Vojta, Phys. Rev. Lett. 91,
170601 (2003)

[34] S. Sachdev, Quantum Phase Transitions (Cambridge University Press, Cambridge, 1999).

[35] A. W. Sandvik, Phys. Rev. B 59, R14 157 (1999); O. F. Syljuåsen and A. W. Sandvik, Phys. Rev. E 66, 046701 (2002).

[36] A. Dorneich and M. Troyer, Phys. Rev. E 64, 066701 (2001). 